\title{
ON THE NUMERICAL SOLUTION FOR THE FRACTIONAL WAVE EQUATION USING LEGENDRE PSEUDOSPECTRAL METHOD
}

\author{
M.M. Khader ${ }^{1}$, N.H. Sweilam² ${ }^{2}$ T.A. Assiri ${ }^{3}$ \\ ${ }^{1}$ Department of Mathematics \\ Faculty of Science \\ Benha University \\ Benha, EGYPT \\ ${ }^{2}$ Department of Mathematics \\ Faculty of Science \\ Cairo University \\ Giza, EGYPT \\ ${ }^{3}$ Department of Mathematics \\ Faculty of Science \\ University of Um-Alqura \\ SAUDI ARABIA
}

\begin{abstract}
Fractional differential equations have recently been applied in various areas of engineering, science, finance, applied mathematics, bio-engineering and others. However, many researchers remain unaware of this field. In this paper, an efficient numerical method for solving the fractional wave equation (FWE) is considered. The fractional derivative is described in the Caputo sense. The method is based upon Legendre approximations. The properties of Legendre polynomials are utilized to reduce FWE to a system of ordinary differential equations, which solved using the finite difference method (FDM). Numerical solutions of FWE are presented and the results are compared with the exact solution.
\end{abstract}

Received: March 7, 2012

(C) 2013 Academic Publications, Ltd. url: www.acadpubl.eu

${ }^{\S}$ Correspondence author 
Key Words: fractional wave equation, Caputo derivative, finite difference method, Legendre pseudospectral method

\section{Introduction}

Ordinary and partial fractional differential equations have been the focus of many studies due to their frequent appearance in various applications in fluid mechanics, viscoelasticity, biology, physics and engineering, see [2]. Consequently, considerable attention has been given to the solutions of fractional differential equations of physical interest. Most fractional differential equations do not have exact solutions, so approximate and numerical techniques (see [3][6]), must be used. Recently, several numerical methods to solve fractional differential equations have been given such as variational iteration method (see [7]), homotopy perturbation method (see [20], [21]), Adomian decomposition method [8], homotopy analysis method [5] and collocation method (see [9]$[12],[18],[22])$.

We present some necessary definitions and mathematical preliminaries of the fractional calculus theory that will be required in the present paper.

Definition 1. The Caputo fractional derivative operator $D^{\alpha}$ of order $\alpha$ is defined in the following form

$$
D^{\alpha} f(x)=\frac{1}{\Gamma(m-\alpha)} \int_{0}^{x} \frac{f^{(m)}(t)}{(x-t)^{\alpha-m+1}} d t, \quad \alpha>0
$$

where $m-1<\alpha \leq m, m \in \mathbb{N}, x>0$. Similar to integer-order differentiation, Caputo fractional derivative operator is a linear operation

$$
D^{\alpha}(\lambda f(x)+\mu g(x))=\lambda D^{\alpha} f(x)+\mu D^{\alpha} g(x),
$$

where $\lambda$ and $\mu$ are constants. For the Caputo's derivative we have [17]

$$
\begin{gathered}
D^{\alpha} C=0, \quad \mathrm{C} \text { is a constant, } \\
D^{\alpha} x^{n}= \begin{cases}0, & \text { for } n \in \mathbb{N}_{0} \text { and } n<\lceil\alpha\rceil ; \\
\frac{\Gamma(n+1)}{\Gamma(n+1-\alpha)} x^{n-\alpha}, & \text { for } n \in \mathbb{N}_{0} \text { and } n \geq\lceil\alpha\rceil .\end{cases}
\end{gathered}
$$

We use the ceiling function $\lceil\alpha\rceil$ to denote the smallest integer greater than or equal to $\alpha$ and $\mathbb{N}_{0}=\{0,1,2, \ldots\}$. Recall that for $\alpha \in \mathbb{N}$, the Caputo differential operator coincides with the usual differential operator of integer order. 
For more details on fractional derivatives definitions and its properties see ([17], [19]).

Our main goal in this paper is concerned with the application of Legendre pseudospectral method to obtain the numerical solution of the fractional wave equation of the form:

$$
\frac{\partial^{2} u(x, t)}{\partial t^{2}}=d(x, t) \frac{\partial^{\alpha} u(x, t)}{\partial x^{\alpha}}+s(x, t),
$$

on a finite domain $a<x<b, 0 \leq t \leq T$ and the parameter $\alpha$ refers to the Caputo fractional order of spatial derivatives with $1<\alpha \leq 2$. The function $s(x, t)$ is a source term.

We also assume the following initial conditions:

$$
u(x, 0)=u^{0}(x), \quad u_{t}(x, 0)=u^{1}(x), \quad a<x<b,
$$

and the Dirichlet boundary conditions

$$
u(a, t)=u(b, t)=0 .
$$

Note that $\alpha=2$, Eq.(3) is the classical wave equation:

$$
\frac{\partial^{2} u(x, t)}{\partial t^{2}}=d(x, t) \frac{\partial^{2} u(x, t)}{\partial x^{2}}+s(x, t) .
$$

Our idea is to apply the Legendre collocation method to discretize (3) to get a linear system of ODEs thus greatly simplifying the problem, and use FDM ([14]-[16]) to solve the resulting system.

Legendre polynomials are well known family of orthogonal polynomials on the interval $[-1,1]$ that have many applications [18]. They are widely used because of their good properties in the approximation of functions. However, with our best knowledge, very little work was done to adapt this polynomials to the solution of fractional differential equations.

The organization of this paper is as follows. In the next section, the approximation of fractional derivative $D^{\alpha} y(x)$ is obtained. Section 3 summarizes the application of Legendre collocation method to the solution of (3). As a result a system of ordinary differential equations is formed and the solution of the considered problem is introduced. In Section 4, some numerical results are given to clarify the proposed method. Also a conclusion is given in Section 5 . 


\section{An Approximate Formula of the Fractional Derivative}

The well known Legendre polynomials are defined on the interval $[-1,1]$ and can be determined with the aid of the following recurrence formula [1]:

$$
L_{k+1}(z)=\frac{2 k+1}{k+1} z L_{k}(z)-\frac{k}{k+1} L_{k-1}(z), \quad k=1,2, \ldots,
$$

where $L_{0}(z)=1$ and $L_{1}(z)=z$. In order to use these polynomials on the interval $[0,1]$ we define the so called shifted Legendre polynomials by introducing the change of variable $z=2 x-1$. Let the shifted Legendre polynomials $L_{k}(2 x-1)$ be denoted by $P_{k}(x)$. Then $P_{k}(x)$ can be obtained as follows

$$
P_{k+1}(x)=\frac{(2 k+1)(2 x-1)}{(k+1)} P_{k}(x)-\frac{k}{k+1} P_{k-1}(x), \quad k=1,2, \ldots,
$$

where $P_{0}(x)=1$ and $P_{1}(x)=2 x-1$. The analytic form of the shifted Legendre polynomials $P_{k}(x)$ of degree $k$ given by

$$
P_{k}(x)=\sum_{i=0}^{k}(-1)^{k+i} \frac{(k+i) ! x^{i}}{(k-i)(i !)^{2}} .
$$

Note that $P_{k}(0)=(-1)^{k}$ and $P_{k}(1)=1$. The orthogonality condition is:

$$
\int_{0}^{1} P_{i}(x) P_{j}(x) d x= \begin{cases}\frac{1}{2 i+1}, & \text { for } i=j \\ 0, & \text { for } i \neq j .\end{cases}
$$

The function $u(x)$, which is square integrable in $[0,1]$, may be expressed in terms of shifted Legendre polynomials as

$$
u(x)=\sum_{i=0}^{\infty} c_{i} P_{i}(x)
$$

where the coefficients $c_{i}$ are given by

$$
c_{i}=(2 i+1) \int_{0}^{1} u(x) P_{i}(x) d x, \quad i=1,2, \ldots .
$$

In practice, only the first $(m+1)$-terms shifted Legendre polynomials are considered. Then we have:

$$
y_{m}(x)=\sum_{i=0}^{m} y_{i} P_{i}(x)
$$

In the following theorem we will approximate the fractional derivative of $y(x)$. 
Theorem 1. Let $u(x)$ be approximated by shifted Legendre polynomials as (11) and also suppose $\alpha>0$ then

$$
D^{\alpha}\left(u_{m}(x)\right)=\sum_{i=\lceil\alpha\rceil}^{m} \sum_{k=\lceil\alpha\rceil}^{i} u_{i} w_{i, k}^{(\alpha)} x^{k-\alpha}
$$

where $w_{i, k}^{(\alpha)}$ is given by

$$
w_{i, k}^{(\alpha)}=\frac{(-1)^{(i+k)}(i+k) !}{(i-k) !(k) ! \Gamma(k+1-\alpha)} .
$$

Proof. Since the Caputo's fractional differentiation is a linear operation we have:

$$
D^{\alpha}\left(u_{m}(x)\right)=\sum_{i=0}^{m} y_{i} D^{\alpha}\left(P_{i}(x)\right)
$$

Employing Eqs.(1)-(2) in Eq.(7) we have

$$
D^{\alpha} P_{i}(x)=0, \quad i=0,1, \ldots,\lceil\alpha\rceil-1, \quad \alpha>0 .
$$

Also, for $i=\lceil\alpha\rceil, \ldots, m$, by using Eqs.(1)-(2) and (7) we get

$$
D^{\alpha} P_{i}(x)=\sum_{k=0}^{i} \frac{(-1)^{i+k}(i+k) !}{(i-k) !(k !)^{2}} D^{\alpha}\left(x^{k}\right)=\sum_{k=\lceil\alpha\rceil}^{i} \frac{(-1)^{i+k}(i+k) !}{(i-k) !(k !) \Gamma(k+1-\alpha)} x^{k-\alpha},
$$

a combination of Eqs.(14), (15) and (16) leads to the desired result.

Test Example. Consider the case when $u(x)=x^{2}$ and $m=2, \alpha=0.5$, the shifted Legendre series of $x^{2}$ is

$$
x^{2}=\frac{1}{3} P_{0}(x)+\frac{1}{2} P_{1}(x)+\frac{1}{6} P_{2}(x) .
$$

Hence

$$
D^{\frac{1}{2}} x^{2}=\sum_{i=1}^{2} \sum_{k=1}^{i} y_{i} w_{i, k}^{\left(\frac{1}{2}\right)} x^{k-\frac{1}{2}}
$$

where

therefore

$$
w_{1,1}^{\left(\frac{1}{2}\right)}=\frac{2}{\Gamma\left(\frac{3}{2}\right)}, w_{2,1}^{\left(\frac{1}{2}\right)}=\frac{-6}{\Gamma\left(\frac{3}{2}\right)}, w_{2,2}^{\left(\frac{1}{2}\right)}=\frac{12}{\Gamma\left(\frac{5}{2}\right)},
$$

$$
D^{\frac{1}{2}} x^{2}=x^{-\frac{1}{2}}\left[y_{1} w_{1,1}^{\left(\frac{1}{2}\right)} x+y_{2} w_{2,1}^{\left(\frac{1}{2}\right)} x+y_{2} w_{2,2}^{\left(\frac{1}{2}\right)} x^{2}\right]=\frac{2}{\Gamma\left(\frac{5}{2}\right)} x^{\frac{3}{2}} .
$$




\section{Procedure Solution of the Fractional Wave Equation}

Consider the fractional wave equation of type given in Eq.(3). In order to use Legendre collocation method, we first approximate $u(x, t)$ as:

$$
u_{m}(x, t)=\sum_{i=0}^{m} u_{i}(t) P_{i}(x)
$$

From Eqs.(3), (17) and Theorem 1 we have

$$
\sum_{i=0}^{m} \frac{d^{2} u_{i}(t)}{d t^{2}} P_{i}(x)=d(x, t) \sum_{i=\lceil\alpha\rceil}^{m} \sum_{k=\lceil\alpha\rceil}^{i} u_{i}(t) w_{i, k}^{(\alpha)} x^{k-\alpha}+s(x, t)
$$

We now collocate Eq.(18) at $(m+1-\lceil\alpha\rceil)$ points $x_{p}, p=0,1, \ldots, m-\lceil\alpha\rceil$ as:

$$
\sum_{i=0}^{m} \ddot{u}_{i}(t) P_{i}\left(x_{p}\right)=d\left(x_{p}, t\right) \sum_{i=\lceil\alpha\rceil}^{m} \sum_{k=\lceil\alpha\rceil}^{i} u_{i}(t) w_{i, k}^{(\alpha)} x_{p}^{k-\alpha}+s\left(x_{p}, t\right) .
$$

For suitable collocation points we use roots of shifted Legendre polynomial $P_{m+1-\lceil\alpha\rceil}(x)$.

Also, by substituting Eqs.(17) and (12) in the boundary conditions (5) we can obtain $\lceil\alpha\rceil$ equations as follows:

$$
\sum_{i=0}^{m}(-1)^{i} u_{i}(t)=0, \quad \sum_{i=0}^{m} u_{i}(t)=0 .
$$

Eq.(19), together with $\lceil\alpha\rceil$ equations of the boundary conditions (20), give $(m+1)$ of ordinary differential equations which can be solved, for the unknowns $u_{i}, i=0,1, \ldots, m$, using the finite difference method, as described in the following section.

\section{Numerical Results}

In this section, we implement the proposed method to solve FWE (3) with $\alpha=1.8$, of the form:

$$
\frac{\partial^{2} u(x, t)}{\partial t^{2}}=d(x, t) \frac{\partial^{1.8} u(x, t)}{\partial x^{1.8}}+s(x, t), \quad 0<x<1, \quad t>0
$$


with the coefficient function: $d(x, t)=\Gamma(1.2) x^{1.8}$, and the source function: $s(x, t)=x^{2}(4 x-1) e^{-t}$, with initial conditions: $u(x, 0)=x^{2}(1-x), \quad u_{t}(x, 0)=$ $x^{2}(x-1)$, and Dirichlet conditions: $u(0, t)=u(1, t)=0$.

The exact solution to this problem is $u(x, t)=x^{2}(1-x) e^{-t}$, which can be verified by applying the fractional differential formula (2).

We apply the proposed method with $m=3$, and approximate the solution as follows

$$
u_{3}(x, t)=\sum_{i=0}^{3} u_{i}(t) P_{i}(x)
$$

Using Eq.(19) we have

$$
\sum_{i=0}^{3} \ddot{u}_{i}(t) P_{i}\left(x_{p}\right)=d\left(x_{p}, t\right) \sum_{i=2}^{3} \sum_{k=2}^{i} u_{i}(t) w_{i, k}^{(1.8)} x_{p}^{k-1.8}+s\left(x_{p}, t\right), \quad p=0,1,
$$

where $x_{p}$ are roots of shifted Legendre polynomial $P_{2}(x)$, i.e.

$$
x_{0}=0.211324, \quad x_{1}=0.788675 .
$$

By using Eqs.(20) and (22) we obtain the following system of ODEs

$$
\begin{gathered}
\ddot{u}_{0}(t)+k_{1} \ddot{u}_{1}(t)+k_{2} \ddot{u}_{3}(t)=R_{1} u_{2}(t)+R_{2} u_{3}(t)+s_{0}(t), \\
\ddot{u}_{0}(t)+k_{11} \ddot{u}_{1}(t)+k_{22} \ddot{u}_{3}(t)=R_{11} u_{2}(t)+R_{22} u_{3}(t)+s_{1}(t), \\
u_{0}(t)-u_{1}(t)+u_{2}(t)-u_{3}(t)=0, \\
u_{0}(t)+u_{1}(t)+u_{2}(t)+u_{3}(t)=0,
\end{gathered}
$$

where

$$
\begin{gathered}
k_{1}=P_{1}\left(x_{0}\right), \quad k_{2}=P_{3}\left(x_{0}\right), \quad k_{11}=P_{1}\left(x_{1}\right), \quad k_{22}=P_{3}\left(x_{1}\right), \\
R_{1}=d\left(x_{0}, t\right) w_{2,2}^{(1.8)} x_{0}^{0.2}, \quad R_{2}=d\left(x_{0}, t\right)\left[w_{3,2}^{(1.8)} x_{0}^{0.2}+w_{3,3}^{(1.8)} x_{0}^{1.2}\right], \\
R_{11}=d\left(x_{1}, t\right) w_{2,2}^{(1.8)} x_{1}^{0.2}, \quad R_{22}=d\left(x_{1}, t\right)\left[w_{3,2}^{(1.8)} x_{1}^{0.2}+w_{3,3}^{(1.8)} x_{1}^{1.2}\right] .
\end{gathered}
$$

Now, to use FDM for solving the system (23)-(26), we will use the following notations: $t_{i}=i \Delta t$ to be the integration time $0 \leq t_{i} \leq T, \Delta t=T / N$, for $i=0,1, \ldots, N$. Define $u_{i}^{n}=u_{i}\left(t_{n}\right), s_{i}^{n}=s_{i}\left(t_{n}\right)$. Then the system (23)-(26), is discretized in time and takes the following form

$$
\frac{u_{0}^{n+1}-2 u_{0}^{n}+u_{0}^{n-1}}{\Delta t^{2}}+k_{1} \frac{u_{1}^{n+1}-2 u_{1}^{n}+u_{1}^{n-1}}{\Delta t^{2}}+k_{2} \frac{u_{3}^{n+1}-2 u_{3}^{n}+u_{3}^{n-1}}{\Delta t^{2}}
$$




$$
\begin{gathered}
=R_{1} u_{2}^{n+1}+R_{2} u_{3}^{n+1}+s_{0}^{n+1}, \\
\frac{u_{0}^{n+1}-2 u_{0}^{n}+u_{0}^{n-1}}{\Delta t^{2}}+k_{11} \frac{u_{1}^{n+1}-2 u_{1}^{n}+u_{1}^{n-1}}{\Delta t^{2}}+k_{22} \frac{u_{3}^{n+1}-2 u_{3}^{n}+u_{3}^{n-1}}{\Delta t^{2}} \\
=R_{11} u_{2}^{n+1}+R_{22} u_{3}^{n+1}+s_{1}^{n+1}, \\
u_{0}^{n+1}-u_{1}^{n+1}+u_{2}^{n+1}-u_{3}^{n+1}=0, \\
u_{0}^{n+1}+u_{1}^{n+1}+u_{2}^{n+1}+u_{3}^{n+1}=0 .
\end{gathered}
$$

We can write the above system (27)-(30) in the following matrix form as follows

$$
\begin{gathered}
\left(\begin{array}{cccc}
1 & k_{1} & -\tau^{2} R_{1} & k_{2}-\tau^{2} R_{2} \\
1 & k_{11} & -\tau^{2} R_{11} & k_{22}-\tau^{2} R_{22} \\
1 & -1 & 1 & -1 \\
1 & 1 & 1 & 1
\end{array}\right)\left(\begin{array}{c}
u_{0}{ }^{n+1} \\
u_{1} \\
u_{2} \\
u_{3}
\end{array}\right) \\
=\left(\begin{array}{cccc}
2 & 2 k_{1} & 0 & 2 k_{2} \\
2 & 2 k_{11} & 0 & 2 k_{22} \\
0 & 0 & 0 & 0 \\
0 & 0 & 0 & 0
\end{array}\right)\left(\begin{array}{c}
u_{0} \\
u_{1} \\
u_{2} \\
u_{3}
\end{array}\right)-\left(\begin{array}{cccc}
1 & k_{1} & 0 & k_{2} \\
1 & k_{11} & 0 & k_{22} \\
0 & 0 & 0 & 0 \\
0 & 0 & 0 & 0
\end{array}\right)\left(\begin{array}{c}
u_{0} \\
u_{1} \\
u_{2} \\
u_{3}
\end{array}\right) \\
\\
\end{gathered}
$$

We use the notation for the above system

$$
\begin{aligned}
& A U^{n+1}=B U^{n}-C U^{n-1}+S^{n+1}, \\
& \text { or } \\
& U^{n+1}=A^{-1} B U^{n}-A^{-1} C U^{n-1}+A^{-1} S^{n+1},
\end{aligned}
$$

where $U^{n}=\left(u_{0}^{n}, u_{1}^{n}, u_{2}^{n}, u_{3}^{n}\right)^{T}$ and $S^{n}=\left(s_{0}^{n}, s_{1}^{n}, 0,0\right)^{T}$.

The obtained numerical results by means of the proposed method are shown in table 1 and figures 1 and 2. In table 1, the absolute errors between the exact solution $u_{e x}$ and approximate solution $u_{\text {approx }}$ at $m=3$ and $m=5$ with the final time $T=2$ are given. Also, in figures 1 and 2, comparison between the exact and approximate solution at $T=1$ with time step $\tau=0.0025$, and $m=3$, $m=5$, respectively, are presented. 


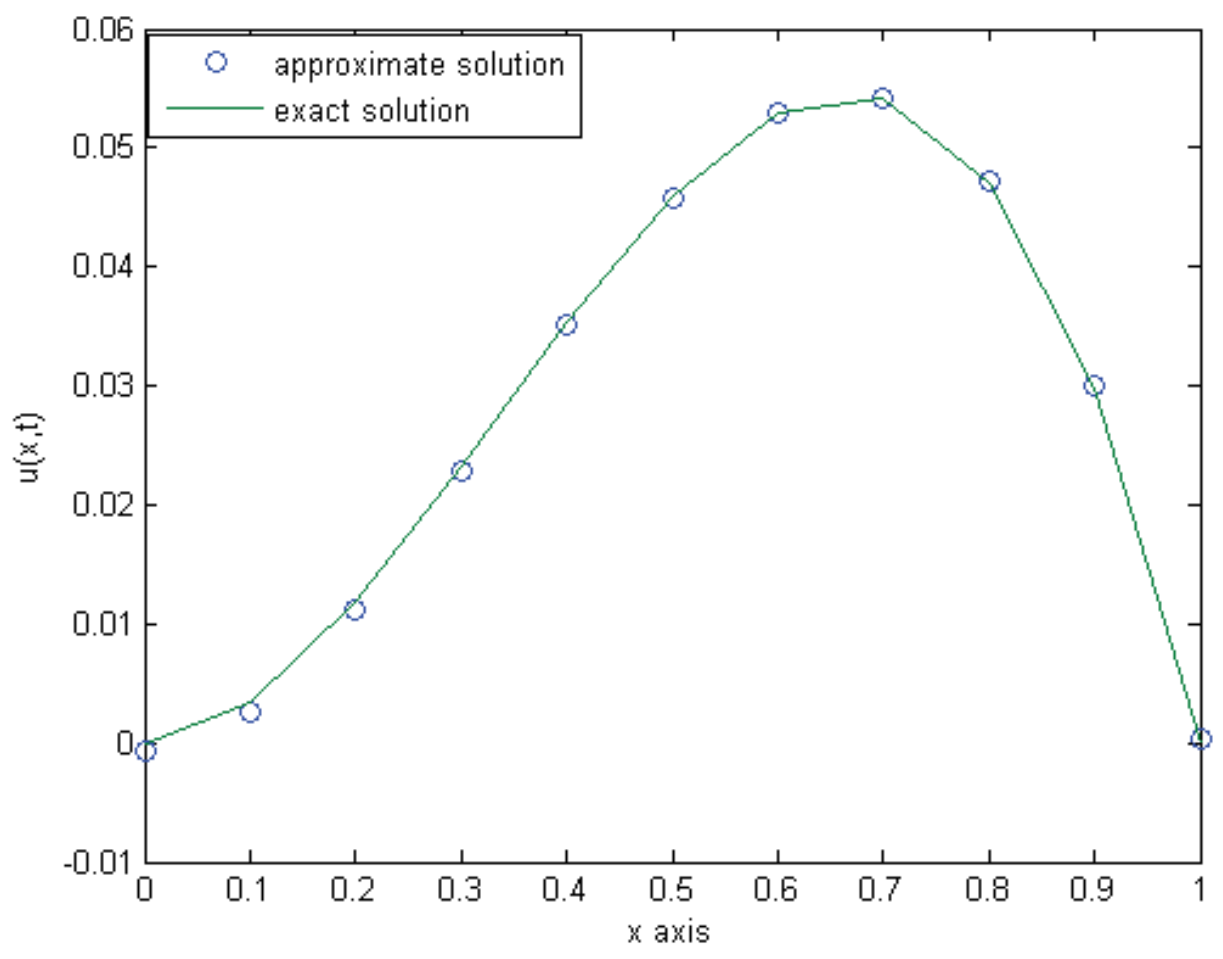

Figure 1: Comparison between the exact and approximate solutions at $T=1$ with $\tau=0.0025, m=3$

\section{Conclusion}

The properties of the Legendre polynomials are used to reduce the fractional wave equation to the solution of system of ordinary differential equations which solved by using FDM. The fractional derivative is considered in the Caputo sense. The solution obtained using the suggested method is in excellent agreement with the already existing ones and show that this approach can be solved the problem effectively. Although we only considered a model problem in this paper, the main idea and the used techniques in this work are also applicable to many other problems. It is evident that the overall errors can be made smaller by adding new terms from the series (17). Comparisons are made be- 


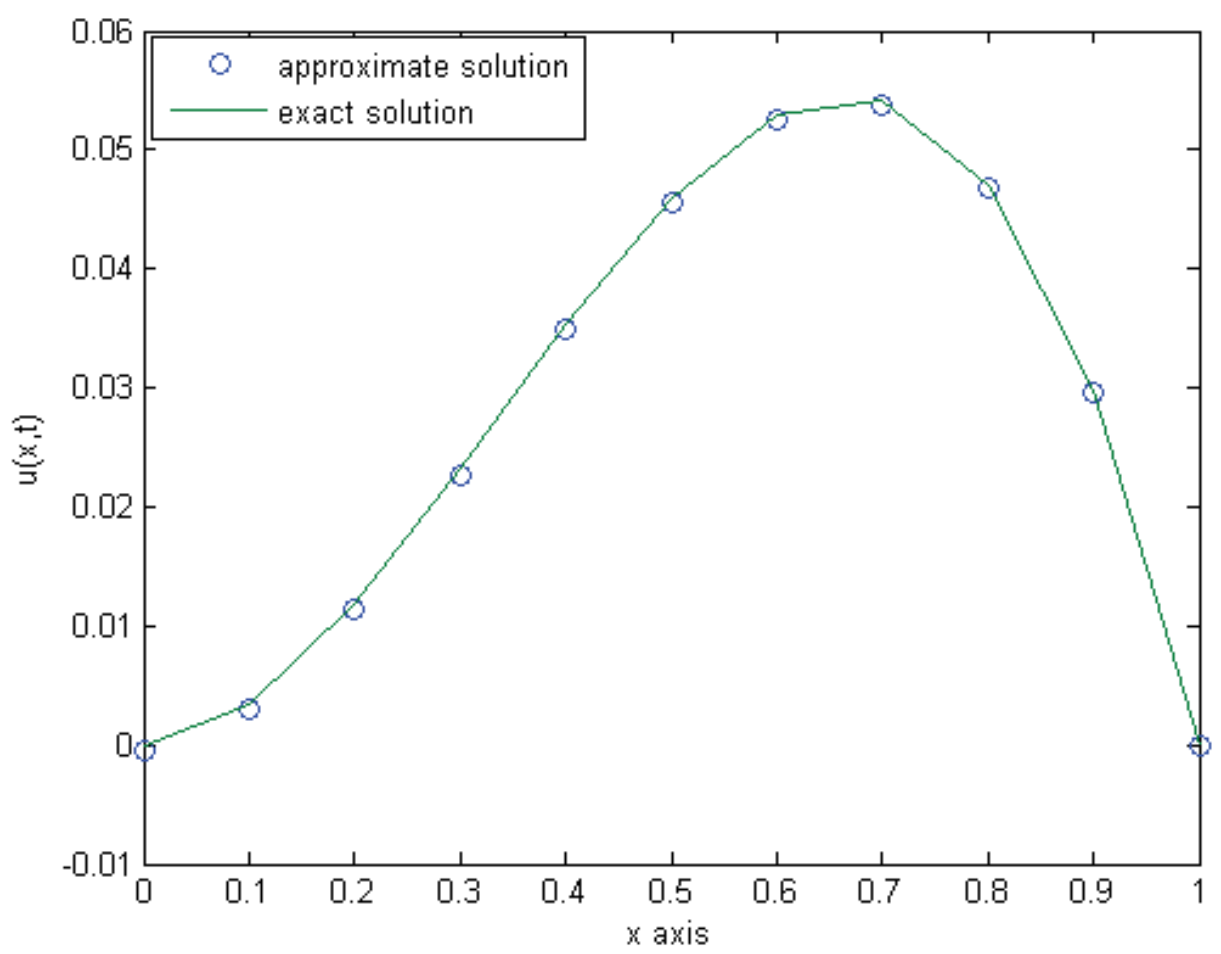

Figure 2: Figure 2. Comparison between the exact and approximate solutions at $T=1$ with $\tau=0.0025, m=5$

tween approximate solutions and exact solutions to illustrate the validity and the great potential of the technique. All computations in this paper are done using Matlab 7.1.

\section{References}

[1] W.W. Bell, Special Functions For Scientists and Engineers, Great Britain, Butler and Tanner Ltd, Frome and London (1968).

[2] S. Das, Functional Fractional Calculus for System Identification and Controls, Springer, New York (2008). 


\begin{tabular}{|l|c|c|}
\hline$x$ & $\left|u_{\text {ex }}-u_{\text {approx }}\right|$ at $m=3$ & $\left|u_{\text {ex }}-u_{\text {approx }}\right|$ at $m=5$ \\
\hline 0.0 & $4.483787 \mathrm{e}-03$ & $2.726496 \mathrm{e}-04$ \\
\hline 0.1 & $4.479660 \mathrm{e}-03$ & $3.455890 \mathrm{e}-04$ \\
\hline 0.2 & $4.201329 \mathrm{e}-03$ & $3.809670 \mathrm{e}-04$ \\
\hline 0.3 & $3.695172 \mathrm{e}-03$ & $3.809103 \mathrm{e}-04$ \\
\hline 0.4 & $3.007566 \mathrm{e}-03$ & $3.514280 \mathrm{e}-04$ \\
\hline 0.5 & $2.184889 \mathrm{e}-03$ & $3.009263 \mathrm{e}-04$ \\
\hline 0.6 & $1.273510 \mathrm{e}-03$ & $2.387121 \mathrm{e}-04$ \\
\hline 0.7 & $0.319831 \mathrm{e}-03$ & $1.735125 \mathrm{e}-04$ \\
\hline 0.8 & $0.629793 \mathrm{e}-03$ & $1.119821 \mathrm{e}-04$ \\
\hline 0.9 & $1.528978 \mathrm{e}-03$ & $0.572150 \mathrm{e}-04$ \\
\hline 1.0 & $2.331347 \mathrm{e}-03$ & $0.072566 \mathrm{e}-04$ \\
\hline
\end{tabular}

Table 1: The absolute error between the exact and approximate solutions at $m=3, m=5$ and $T=2$

[3] K. Diethelm, An algorithm for the numerical solution of differential equations of fractional order, Electron. Trans. Numer. Anal., 5 (1997).

[4] M. Enelund, B.L. Josefson, Time-domain finite element analysis of viscoelastic structures with fractional derivatives constitutive relations, AIAA J., 35, No. 10 (1997), 1630-1637.

[5] I. Hashim, O. Abdulaziz, S. Momani, Homotopy analysis method for fractional IVPs, Commun. Nonlinear Sci. Numer. Simul., 14 (2009), 674-684.

[6] J.H. He, Approximate analytical solution for seepage flow with fractional derivatives in porous media, Computer Methods in Applied Mechanics and Engineering, 167, No. 1-2 (1998), 57-68.

[7] M. Inc, The approximate and exact solutions of the space-and timefractional Burger's equations with initial conditions by variational iteration method, J. Math. Anal. Appl., 345 (2008), 476-484.

[8] H. Jafari, V. Daftardar-Gejji, Solving linear and nonlinear fractional diffusion and wave equations by Adomian decomposition method, Appl. Math. and Comput., 180 (2006), 488-497. 
[9] M.M. Khader, On the numerical solutions for the fractional diffusion equation, Communications in Nonlinear Science and Numerical Simulations, 16 (2011), 2535-2542.

[10] M.M. Khader, N.H. Sweilam, A.M. S. Mahdy, An efficient numerical method for solving the fractional diffusion equation, J. of Applied Mathematics and Bioinformatics, 1 (2011), 1-12.

[11] M.M. Khader, A.S. Hendy, The approximate and exact solutions of the fractional-order delay differential equations using Legendre pseudospectral method, International Journal of Pure and Applied Mathematics, 74, No. 3 (2012), 287-297.

[12] M.M. Khader, Introducing an efficient modification of the homotopy perturbation method by using Chebyshev polynomials, Arab Journal of Mathematical Sciences, 18 (2012), 61-71.

[13] Kreyszig E. Introductory Functional Analysis with Applications, John Wiley and sons, Inc. (1978).

[14] Ch. Lubich, Discretized fractional calculus, SIAM J. Math. Anal., 17 (1986), 704-719.

[15] M.M. Meerschaert, C. Tadjeran, Finite difference approximations for fractional advection-dispersion flow equations, J. Comput. Appl. Math. 172, No. 1 (2004), 65-77.

[16] M.M. Meerschaert, C. Tadjeran, Finite difference approximations for twosided space-fractional partial differential equations, Appl. Numer. Math. 56 (2006), 80-90.

[17] I. Podlubny, Fractional Differential Equations, Academic Press, New York (1999).

[18] E.A. Rawashdeh, Numerical solution of fractional integro-differential equations by collocation method, Appl. Math. Comput., 176 (2006), 1-6.

[19] S. Samko, A. Kilbas, O. Marichev, Fractional Integrals and Derivatives: Theory and Applications, Gordon and Breach, London (1993).

[20] N.H. Sweilam, M.M. Khader, R.F. Al-Bar, Numerical studies for a multiorder fractional differential equation, Physics Letters A, 371 (2007), 26-33. 
[21] N.H. Sweilam, M.M. Khader, R.F. Al-Bar, Homotopy perturbation method for linear and nonlinear system of fractional integro-differential equations, International Journal of Computational Mathematics and Numerical Simulation, 1, No. 1 (2008), 73-87.

[22] N.H. Sweilam, M.M. Khader, A Chebyshev pseudo-spectral method for solving fractional integro-differential equations, ANZIAM, 51 (2010), 464475 .

[23] N.H. Sweilam, M.M. Khader, A.M. Nagy, Numerical solution of two-sided space-fractional wave equation using finite difference method, Journal of Computional and Applied Mathematics, 235 (2011), 2832-2841.

[24] N.H. Sweilam, M.M. Khader, A.M.S. Mahdy, Crank-Nicolson finite difference method for solving time-fractional diffusion equation, Journal of Fractional Calculus and Applications, 2, No. 2 (2012), 1-9. 
